\title{
Hedgehog Stimulation Suppresses Clonogenicity and Activates NOTCH Signalling in T-lymphoblastic Leukaemia Jurkat Cells
}

\author{
YUKI OKUHASHI $^{1,2}$, MAI ITOH ${ }^{1}$ and SHUJI TOHDA ${ }^{1}$ \\ ${ }^{I}$ Department of Laboratory Medicine, Tokyo Medical and Dental University, Tokyo, Japan; \\ ${ }^{2}$ Department of Medical Technology, Tokyo University of Technology, Tokyo, Japan
}

\begin{abstract}
Background/Aim: Hedgehog $(\mathrm{HH})$ and NOTCH pathways are involved in the regulation of cancer stem cells and haematopoietic malignancies. However, the effects of HH stimulation on cell growth and NOTCH signalling in acute T-lymphoblastic leukaemia (T-ALL) cells have not been elucidated. Materials and Methods: Two T-ALL cell lines, Jurkat and KOPT-K1 harbouring activating NOTCHI mutations, were cultured with recombinant Sonic (S) HH and analysed for proliferation, colony formation, and expression of NOTCH-regulated genes and proteins. Results: SHH stimulation did not affect cell growth but suppressed colony formation, increased the levels of cleaved NOTCH1 fragment characteristic for NOTCH1 activation, and upregulated mRNA expression of HES1, while decreasing that of MYC in Jurkat cells. However, no such effects were observed in KOPT-K1 cells. Conclusion: Our results indicate that SHH stimulation activates NOTCH signalling in Jurkat cells, thus disclosing a novel relationship between $\mathrm{HH}$ and NOTCH pathways.
\end{abstract}

Hedgehog $(\mathrm{HH})$ proteins mediate intercellular signalling processes regulating haematopoietic stem cells and development of various cancers such as glioma, breast cancer, leukaemia, and lymphoma (1). The binding of $\mathrm{HH}$ proteins to their cognate receptor Patched-1 (PTCH1) releases Smoothened (SMO) which induces nuclear translocation of glioma-associated oncogene homolog (GLI) and upregulates transcription of various target genes (2-4). Among the three mammalian $\mathrm{HH}$ homologs, Sonic $\mathrm{HH}$ (SHH), Indian HH, and Desert HH, SHH plays an important role in haematopoiesis and immunity $(1,5)$.

Correspondence to: Dr. Shuji Tohda, Department of Laboratory Medicine, Tokyo Medical and Dental University, Yushima 1-5-45, Bunkyo-Ku, Tokyo 113-8519, Japan. Tel: +81 358035334, Fax: +81 358035629, e-mail: tohda.mlab@tmd.ac.jp

Key Words: Hedgehog, NOTCH, MYC, leukaemia.
We have previously reported that SHH had no effect on the short-term growth of acute myeloid leukaemia (AML) cells but promoted clonogenic cell recovery (CCR) after culture in suspension, suggesting that $\mathrm{SHH}$ regulates the capacity for self-renewal of leukaemia stem cells or progenitor cells present in the AML cell population (6). However, the effects of SHH stimulation on acute T-lymphoblastic leukaemia (TALL) cells are not fully elucidated.

The highly conserved NOTCH signalling system controls multiple cellular processes, including expansion of haematopoietic stem cells and leukaemia cells. In normal haematopoietic cells, transmembrane NOTCH receptors are cleaved following ligand binding, and the cleaved fragments are translocated into the nucleus where they regulate the expression of the target genes coding for transcription factors HES1 and MYC, and transcriptional regulator DTX1 (7). Constitutive activation of NOTCH signalling is observed in T-ALL patients who carry activating mutations in the NOTCHl gene (8).

These data indicate that both $\mathrm{HH}$ and $\mathrm{NOTCH}$ signalling pathways are involved in leukaemia-related processes, suggesting their possible interaction, which, however, remains poorly understood. Although $\mathrm{NOTCH}$ activation upregulates SHH protein expression in stem cells of the central nervous system (9) and its inhibition downregulates GLI1 expression in retinal progenitor cells (10), the reverse, i.e., the effects of $\mathrm{HH}$ activation on $\mathrm{NOTCH}$ signalling are unknown.

In this study, we investigated the impact of SHH stimulation on the growth of and NOTCH signalling in T-ALL cell lines. This is the first study investigating the relationship between HH and NOTCH pathways in human T-ALL cells.

\section{Materials and Methods}

Cells and reagents. Two human T-ALL cell lines, Jurkat and KOPT$\mathrm{K} 1$, harbouring activating $\mathrm{NOTCH} 1$ mutations were donated by Dr. Harashima and Dr. Orita (Fujisaki Cell Centre, Okayama, Japan). Recombinant SHH was purchased from R\&D Systems (Minneapolis, MN, USA). Three gamma-secretase inhibitors (GSIs), 

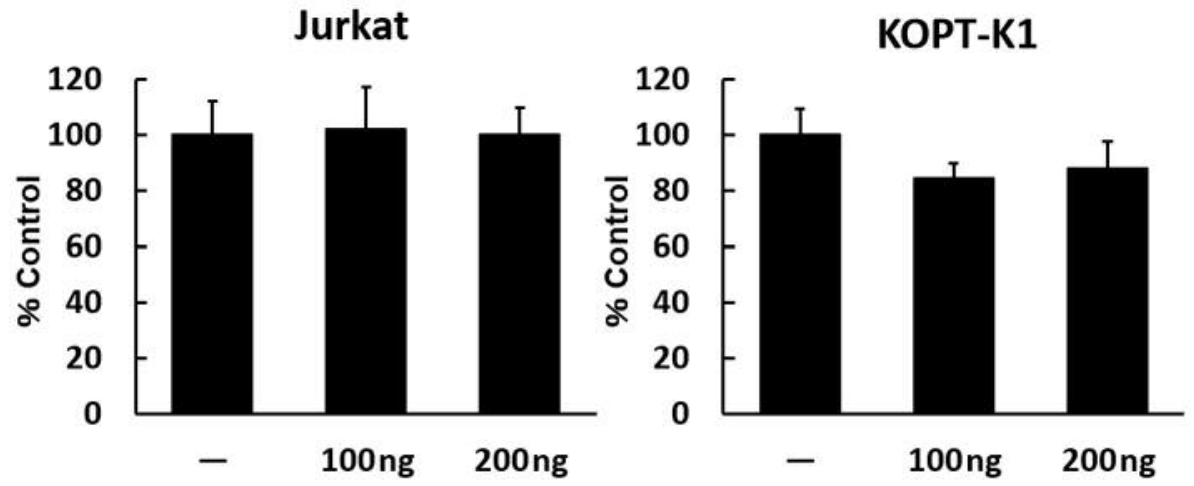

Figure 1. Effects of SHH on the growth of Jurkat cells (left panel) and KOPT-K1 cells (right panel). Cells were cultured with the indicated concentrations of SHH for 3 days, and their growth was examined using a colorimetric assay and expressed as the percentage (mean \pm standard deviation) of control cells cultured without SHH.

GSI-IX, GSI-XII, and GSI-XXI were obtained from Calbiochem (San Diego, CA, USA) and dissolved in dimethyl sulfoxide (DMSO) before use.

Short-term growth assay. SHH effects on cell growth were examined by a colorimetric WST-8 assay (Dojindo Laboratories, Kumamoto, Japan). Cells $\left(5 \times 10^{4}\right.$ per well) were cultured in RPMI1640 medium containing 10\% fetal calf serum (FCS) (Gibco, Invitrogen, Carlsbad, CA, USA) and different concentrations of SHH for 3 days. Then, the WST-8 reagent was added and the optical density (OD) was measured using an ELISA plate reader. Cell growth was calculated as the percentage of the mean OD value for control cells cultured without SHH.

Colony formation assay. Cells were seeded in 96-well culture plates (200 cells/well) in RPMI-1640 containing $0.8 \%$ methylcellulose and $10 \%$ FCS with or without SHH or GSIs for 7 days. The number of colonies containing more than 20 cells was determined under an inverted microscope.

Self-renewal capacity. Cell self-renewal was evaluated by the recovery of clonogenic cells cultured in suspension (11). T-ALL cells $\left(1 \times 10^{5}\right)$ maintained in $1 \mathrm{ml}$ RPMI-1640 with or without SHH $(200 \mathrm{ng} / \mathrm{ml})$ for 3 days were harvested, counted, and plated (200 cells/well) in the medium containing $0.8 \%$ methylcellulose and $10 \%$ FCS. Colony numbers were counted after 7 days and the plating efficiency after suspension culture (PEs) was expressed as the ratio of the mean number of colonies to that of plated cells. Then, clonogenic cells recovery (CCR) was calculated by multiplying the number of harvested cells by the PEs.

Immunoblotting. NOTCH activation was examined by immunoblotting. Cells cultured with or without $200 \mathrm{ng} / \mathrm{ml} \mathrm{SHH}$ for $48 \mathrm{~h}$ were harvested and lysed; then, cell lysates equivalent to $1 \times 10^{6}$ cells/lane were subjected to sodium dodecyl sulfatepolyacrylamide gel electrophoresis (SDS-PAGE). The separated proteins were tested for the reaction with the following antibodies: anti-cleaved NOTCH1 (Val1744) (Cell Signaling Technology, Danvers, MA, USA) to detect the intracellular NOTCH1 fragment, anti-NOTCH1 (Santa Cruz Biotechnology,
Table I. Effects of SHH stimulation on clonogenic cell recovery of Jurkat cells after suspension culture.

\begin{tabular}{lccccc}
\hline \multicolumn{2}{c}{ Cell no. $\left(\times 10^{5}\right)$} & \multicolumn{2}{c}{ PEs $\left(\times 10^{-2}\right)$} & \multicolumn{2}{c}{ CCR $\left(\times 10^{4}\right)$} \\
\hline Control & SHH & Control & SHH & Control & SHH \\
\hline $4.0 \pm 0.8$ & $5.0 \pm 0.6$ & $22.0 \pm 2.0$ & $7.8 \pm 1.0^{*}$ & $8.8 \pm 2.0$ & $3.9 \pm 1.0^{*}$ \\
\hline
\end{tabular}

Cell no.: Number of cells harvested from suspension culture; PEs: plating efficiency after suspension culture; CCR: clonogenic cells recovery. ${ }^{*} p<0.05$ versus control.

CA, USA), and anti- $\alpha$-tubulin (Abcam, Cambridge, MA, USA) to ensure equal loading.

Quantitative RT-PCR. Gene expression was examined by quantitative RT-PCR. RNA was extracted from cells treated with $200 \mathrm{ng} / \mathrm{ml} \mathrm{SHH}$ for $24 \mathrm{~h}$ and used for first-strand cDNA synthesis. Quantitative RT-PCR was performed in a LightCycler (Roche Diagnostics, Manheim, Germany) using QuantiTect primers (QIAGEN, Hilden, Germany), LightCycler primer sets, and a FastStart DNA Master SYBR Green I kit (Roche Diagnostics, Manheim, Germany)). The expression level of each mRNA was normalized to that of $\beta$-actin-encoding mRNA.

\section{Results}

SHH stimulation did not exert any significant effect on the short-term growth of Jurkat and KOPT-K1 cells (Figure 1), it decreased the colony number and colony size of Jurkat cells compared to untreated control (Figure 2). CCR, which reflects the self-renewal capacity of leukaemia stem cells, was inhibited by SHH stimulation in Jurkat cells (Table I). Treatment with GSIs used as NOTCH inhibitors also suppressed colony formation by Jurkat cells (Figure 3). 


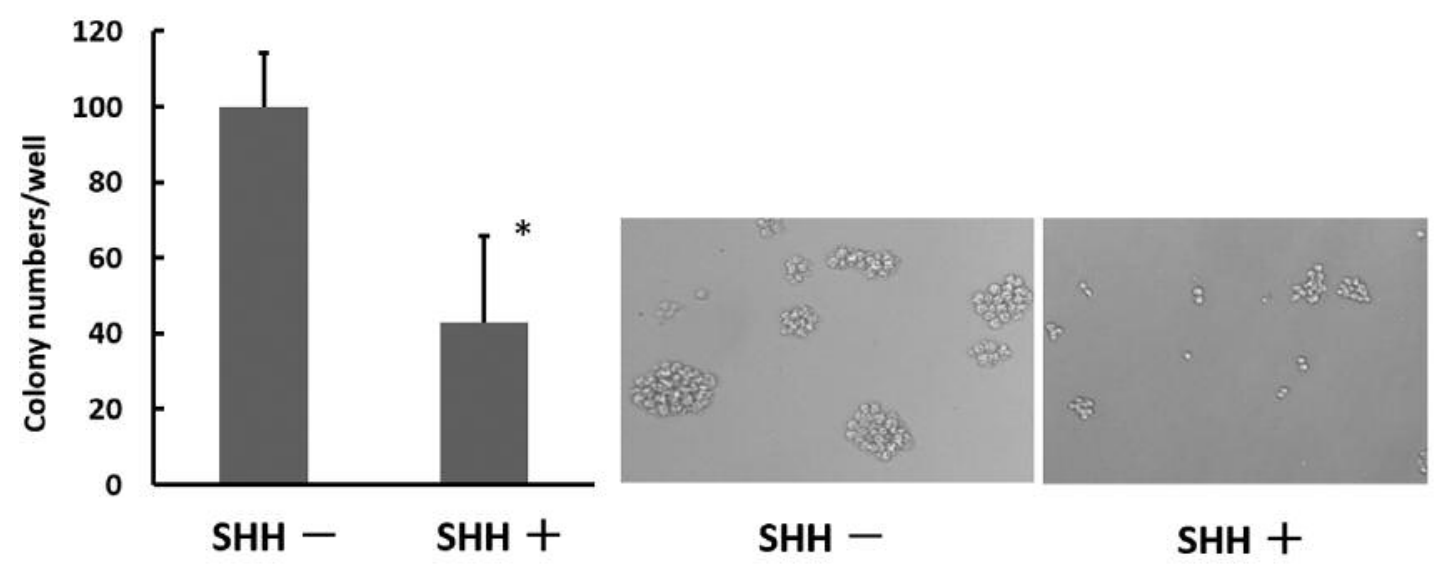

Figure 2. Effects of SHH stimulation on colony formation by Jurkat cells. Cells were cultured with or without SHH (200 ng/ml) for 7 days, and colony numbers were counted under an inverted microscope (left panel). Representative images of cell colonies are shown on the right panel. The data are expressed as the mean \pm standard deviation $(* p<0.05)$.

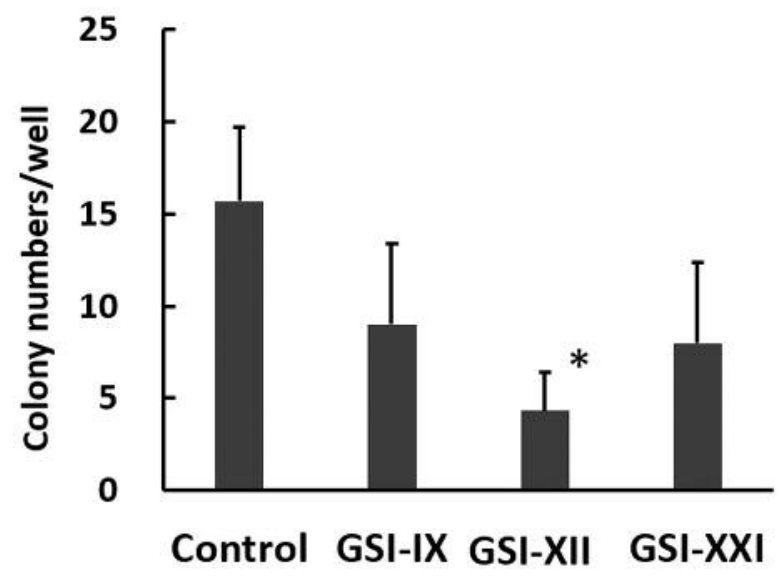

Figure 3. Effects of GSIs on colony formation by Jurkat cells. Cells were cultured with $6 \mu M$ GSIs for 7 days and colony numbers were counted. The data are expressed as the mean \pm standard deviation $(* p<0.05$ versus control).

However, the colony assay could not be performed for KOPT$\mathrm{K} 1$ cells because they proliferated diffusely in methylcellulose, and the exact number of colonies could not be counted.

In addition, $\mathrm{SHH}$ stimulation increased the amount of the cleaved NOTCH1 fragment without increasing the total NOTCH1 protein in Jurkat cells; however no significant effect was observed in KOPT-K1 cells (Figure 4).

Finally, SHH stimulation upregulated the expression of HES1 and DTX1 but down-regulated that of MYC in Jurkat cells (Table II). Similar to protein expression, there was no

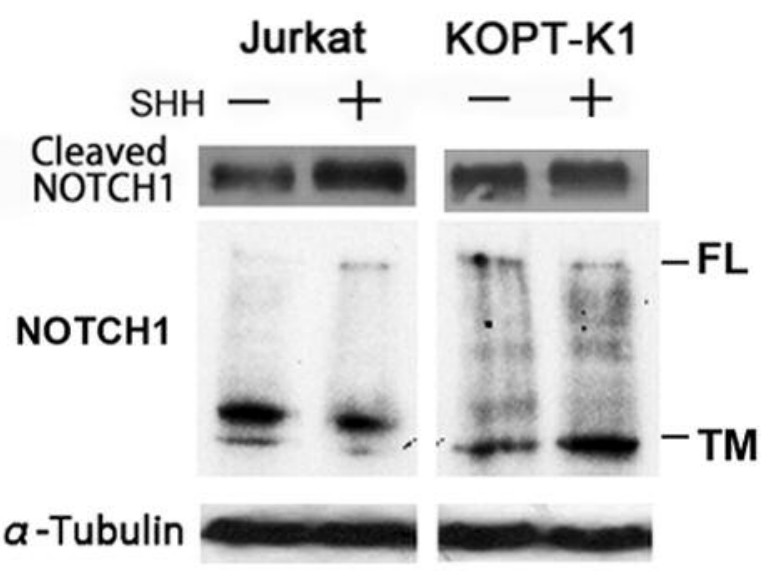

Figure 4. Effects of SHH stimulation on the expression of NOTCH proteins. Cells cultured with or without SHH $(200 \mathrm{ng} / \mathrm{ml})$ for $48 \mathrm{~h}$ were analysed by immunoblotting with antibodies against cleaved NOTCH1, total NOTCH1, and a-tubulin. FL: Full-length NOTCH1; TM: transmembrane NOTCH1 subunit.

difference in gene expression between KOPT-K1 cells treated or not with $\mathrm{SHH}$.

\section{Discussion}

Our findings indicate that $\mathrm{SHH}$ stimulation suppressed colony formation (Figure 2) while activating NOTCH signalling in Jurkat cells (Figure 5). NOTCH activation was confirmed by the increase in the cleaved NOTCH1 fragment and upregulation of HESI mRNA expression. Given that 
Table II. Effects of SHH stimulation on mRNA expression in T-ALL cells.

\begin{tabular}{lcccc}
\hline Cell line & Treatment & HES1 & MYC & DTX1 \\
\hline Jurkat & Control & $1.3 \mathrm{E}-02$ & $6.9 \mathrm{E}-07$ & $1.6 \mathrm{E}-03$ \\
& SHH+ & $9.2 \mathrm{E}-02$ & $2.2 \mathrm{E}-07$ & $1.1 \mathrm{E}-02$ \\
KOPT-K1 & Control & $1.6 \mathrm{E}-02$ & $1.3 \mathrm{E}-06$ & $1.7 \mathrm{E}-01$ \\
& SHH+ & $2.2 \mathrm{E}-02$ & $1.4 \mathrm{E}-06$ & $2.3 \mathrm{E}-01$ \\
\hline
\end{tabular}

Relative mRNA expression was calculated after normalization to $\beta$ actin-encoding mRNA levels.

NOTCH inhibitors decreased colony formation by Jurkat cells (Figure 3), these results suggest that SHH suppressed colony formation through NOTCH-independent mechanisms. It is known that activation of both $\mathrm{HH}$ and $\mathrm{NOTCH}$ signalling upregulates $M Y C$ expression $(2,7)$. However, in our experiments, $M Y C$ expression was downregulated in SHH-stimulated Jurkat cells, which can be the cause of colony formation inhibition, although the mechanisms underlying $M Y C$ down-regulation need to be elucidated.

On the other hand, SHH stimulation had no effect on colony formation and NOTCH activation in KOPT-K1 cells. We have previously reported that an $\mathrm{HH}$ inhibitor cyclopamine suppressed the growth of KOPT-K1 cells but caused no effect on Jurkat cells (12), suggesting that HH signalling is constitutively activated in KOPT-K1 cells. Therefore, SHH stimulation did not affect KOPT-K1 cell growth, whereas the HH inhibitor suppressed it. In contrast, $\mathrm{HH}$-dependent signalling may be inactivated in Jurkat cells, explaining why $\mathrm{SHH}$ regulated colony formation but $\mathrm{HH}$ inhibition did not influence cell proliferation.

In our earlier studies, we showed that NOTCH inhibition by GSIs or siRNA suppressed the growth of KOPT-K1 cells but not Jurkat cells $(13,14)$. Although both cell lines have activating NOTCH1 mutations, Jurkat cells may possess certain unique mechanisms regulating $\mathrm{NOTCH}$ signalling and cross-talk between NOTCH and HH pathways, which differ from those existing in KOPT-K1 cells. The molecular mechanisms underlying $\mathrm{HH}$-dependent activation of $\mathrm{NOTCH}$ in Jurkat cells remain to be elucidated. It should also be clarified whether the processes observed in Jurkat cells occur in other T-ALL cells.

Future studies investigating the cross-talk among HH, $\mathrm{NOTCH}$, and other stemness-related pathways in T-ALL cells would further development of new targeted drugs for the treatment of haematopoietic malignancies.

\section{Acknowledgements}

This work was supported in part by a Grant-in-Aid for Young Scientists (B) from the Japan Society for the Promotion of Science (No. 16K19205).

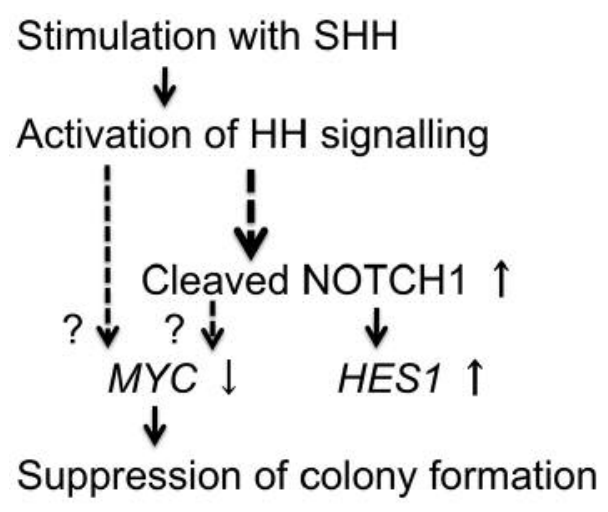

Figure 5. Schematic representation of possible mechanisms regulating colony formation in Jurkat cells.

\section{References}

1 Bai LY, Chiu CF, Lin CW, Hsu NY, Lin CL, Lo WJ and Kao MC: Differential expression of Sonic hedgehog and Gli1 in hematological malignancies. Leukemia 22: 226-228, 2007.

2 Lee J, Platt KA, Censullo P, Ruiz I and Altaba A: Gli1 is a target of Sonic hedgehog that induces ventral neural tube development. Development 124: 2537-2552, 1997.

3 Chen JK, Taipale J, Cooper MK and Beachy PA: Inhibition of Hedgehog signaling by direct binding of cyclopamine to Smoothened. Genes Dev 16: 2743-2748, 2002.

4 Jia Y, Wang Y and Xie J: The Hedgehog pathway: role in cell differentiation, polarity and proliferation. Arch Toxicol 89: 179191, 2015.

5 Bhardwaj G, Murdoch B, Wu D, Baker DP, Williams KP, Chadwick K, Ling LE, Karanu FN and Bhatia M: Sonic hedgehog induces the proliferation of primitive human hematopoietic cells via BMP regulation. Nat Immunol 2: 142143, 2001.

6 Kawaguchi-Ihara N, Okuhashi Y, Itoh M, Murohashi I, Nara N and Tohda S: Promotion of the self-renewal capacity of human leukemia cells by sonic hedgehog protein. Anticancer Res 31: 781-784, 2011.

7 Suzuki T and Chiba S: NOTCH signaling in hematopoietic stem cells. Int J Hematol 82: 285-294, 2005.

8 Weng AP, Ferrando AA, Lee W, Morris JP 4th, Silverman LB, Sanchez-Irizarry C, Blacklow SC, Look AT and Aster JC: Activating mutations of NOTCH1 in human $\mathrm{T}$ cell acute lymphoblastic leukemia. Science 306: 269-271, 2004.

9 Androutsellis-Theotokis A, Leker RR, Soldner F, Hoeppner DJ, Ravin R, Poser SW, Rueger MA, Bae SK, Kittappa R and McKay RD: Notch signalling regulates stem cell numbers in vitro and in vivo. Nature 442: 823-826, 2006.

10 Ringuette R, Atkins M, Lagali PS, Bassett EA, Campbell C, Mazerolle C, Mears AJ, Picketts DJ and Wallace VA: A NotchGli2 axis sustains Hedgehog responsiveness of neural progenitors and Müller glia. Dev Biol 411: 85-100, 2016.

11 Tohda S, Yang GS, Ashman LK, McCulloch EA and Minden MD: Relationship between c-kit expression and proliferation in acute myeloblastic leukemia cell lines. J Cell Physiol 154: 410$418,1993$. 
12 Kawahara T, Kawaguchi-Ihara N, Okuhashi Y, Itoh M, Nara N and Tohda S: Cyclopamine and quercetin suppress the growth of leukemia and lymphoma cells. Anticancer Res 29: 4629-4632, 2009.

13 Okuhashi Y, Nara $\mathrm{N}$ and Tohda S: Effects of $\gamma$-secretase inhibitors on the growth of leukemia cells. Anticancer Res 30: 495-498, 2010.
14 Okuhashi Y, Itoh M, Nara N and Tohda S: NOTCH knockdown affects the proliferation and mTOR signaling of leukemia cells. Anticancer Res 33: 4293-4298, 2013.

Received July 19, 2017

Revised July 31, 2017

Accepted August 1, 2017 